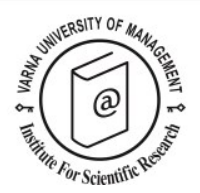

\title{
Examining experience economy approaches to tourists' anticipated experiences: Mainland Chinese travellers consider Australia
}

\author{
Amy M. Osmond ${ }^{1}$, Tingzhen Chen $^{2}$ and Philip L. Pearce ${ }^{3 *}$
}

Received: 07/04/2014 Accepted: 28/08/2014

\footnotetext{
${ }^{1} \mathrm{PhD}$ candidate in Tourism, College of Business, Law and Governance, James Cook University, Townsville, Australia. Email: amy.osmond@my.jcu.edu.au

${ }^{2}$ Postdoctoral researcher, College of Business, Law and Governance, James Cook University, Townsville, Australia. Email: tingzhen.chen@jcu.edu.au

${ }^{3}$ Foundation Professor of tourism, College of Business, Law and Governance, James Cook University, 1 James Cook Drive, Townsville, Queensland, Australia, 4811. Phone: +617 4781 4762, fax: +617 4781 4019, email: philip.pearce@jcu.edu.au

* Corresponding author
}

\begin{abstract}
The present study seeks to explore and compare the perceived importance of Pine and Gilmore's experience realms and Schmitt's categorisations of experience in a tourism context. These approaches to experiences are chosen because they are widely cited, yet rarely compared. The empirical component of the work is based on Chinese tourists who were asked to anticipate their experiences in Australia. Would be travellers in the regions of Guangzhou, Shanghai and Beijing, accessed through an extensive snowball sampling procedure, responded to a detailed and literature-informed set of activity and experience items in a questionnaire format. The study provided evidence that the systems can be seen as joint contributors to understanding experience; the Pine and Gilmore work offers a more holistic appraisal and the Schmitt system presents a more finegrained rendition of the desired experiences. Aesthetic and sensory experiences were shown to be of the highest importance. Experience analysis offers insights and the approach in the study develops a pathway to pursue empirical studies so that researchers can provide benefits for marketers in tourism.
\end{abstract}

(C) 2015 Varna University of Management. All rights reserved

Keywords: Experience economy; experience analysis; Chinese tourists; anticipation; Australia; comparative systems

Citation: Osmond, A., T. Chen and P. Pearce (2015) Examining experience economy approaches to tourists' anticipated experiences: Mainland Chinese travellers consider Australia. European Journal of Tourism Research 10, pp. 95-108

\section{Introduction}

The analysis offered in this research attempts to provide a structured and literature embedded approach to the analysis of expectations. It employs formative ideas from research on the experience economy and provides an analysis 
of anticipated experiences by comparing two approaches for assessing experiences. The specific work which forms the conceptual heart of the study lies in the categories for understanding experience developed by Pine and Gilmore (1999) and alternatively by Schmitt (2003). These approaches to understanding desired experiences are chosen because they are widely cited, yet rarely compared. The work of Pine and Gilmore $(1998,1999)$ has now been cited over 5,400 times while Schmitt's contributions in 1999 and 2003 have over 2,300 citations (Google Scholar, March $\left.29^{\text {th }} 2014\right)$. It is a pivotal aim of this study to apply the categories of experience developed by these well cited researchers to tourists' anticipated experiences and assess the viability of the applications and their special properties and insights. Furthermore, this work seeks to strengthen these widely influential experience schemes through linking them in a tourism context, which to date, has seldom been explored.

The illustrative case for the comparison of anticipated experiences is that of mainland Chinese tourists considering a holiday trip to Australia. The specific sample used to collect the information was generated from three important tourist generating regions in eastern China. This analysis of the various components of anticipated Australian experiences can potentially explore the ways in which two prominent categorisation schemes to assess experiences are related and, further, such an approach may provide insights into the complexities of the Chinese market. Huang and Gross (2010) observe that studies of outbound Chinese tourists are still limited. Much remains to be done beyond the recording and requoting of the numbers and demand (anticipated arrivals).

Following these directions, this study employs a pre-travel psychological profiling approach to analyse in some detail the anticipated features of the Australian experience. In particular, the perceived level of importance attributed by mainland Chinese residents to various components of the experience is examined through a cross-analysis of two different approaches for assessing experiences. This indepth exploration of the Chinese tourists' anticipated Australian experiences provides an appreciation of the complex cultural wants and needs of the market, which is increasingly valued by Chinese visitors (Li, Lai, Harrill, Kline \& Wang, 2011). The work attempts to extend previous studies on Chinese tourists experiences, which the authors argue have tended to take an after-experience approach and reported results built on importance and satisfaction levels cross-referenced by demographic/psychographic factors. Whilst this study explores the experiences for one pairing of continents, the principles underlying experience and used in this analysis arguably have implications for many other pairs of source and destination countries.

\section{Literature review}

This review is structured into two parts. It first considers the growing prominence of the Chinese outbound market and the necessity for intensive analyses on their desired tourist experiences. Then the focus shifts towards highlighting previous research on Chinese tourists' experiences through the use of two experience analysis systems. Schmitt's (2003) categorisation of experiences and Pine and Gilmore's (1999) realms of experiences are both widely cited, however, they are yet to be linked in a tourism context. Furthermore, the cross-analysis of these two systems presents a novel direction of study which attempts to provide an in-depth, multi-dimensional perspective on Chinese tourists' anticipated Australian travel experiences.

China outbound tourism has blossomed in the last decade. For all the countries which seek to attract Chinese visitors it is becoming increasingly important to understand the characteristics of the Chinese market, not only to compete against rival destinations but also to manage issues of tourist well-being, community acceptance and sustainability. In the case of Australia, which was granted Approved Destination Status (ADS) in 1999, the Chinese market has become a key part of the overall tourism industry (DITR, 2007, cited in Weiler \& $\mathrm{Yu}, 2008)$. Chinese visitors now number nearly 800,000 annually (Tourism Research Australia, 2012). The growing size of the China outbound market, including predictions for 100 million outbound travellers by 2020 , indicates its 
importance, and prompts the need for intensive analyses as the market changes (Ai-Ping, 2009; Breakey, Ding \& Lee, 2008).Australia, in common with other western destinations needs to gain a clearer and fuller comprehension of the culturally different Chinese tourists if it is to remain competitive in attracting one of the world's most desired markets (Chow \& Murphy, 2008; Wang \& Davidson, 2010).

Many Chinese residents can now afford the basic necessities of life due to widespread increases in disposable income (Zhou, King \& Turner, 1998). Large numbers of Chinese are now purchasing more luxury items and outbound travel is included in the set of desired consumption experiences. The increasing international travel experience is starting to change the Chinese tourists' demands. In particular, Chinese travellers now expect the host destination and community to have some cultural understanding of their wants and needs (Li et al., 2011).It has also been suggested that Chinese tourists are seeking more in-depth experiences that focus on their inner needs (AiPing, 2009; Xie \& Li, 2009).

Australia, as a tourist destination, has the potential to offer a unique and memorable experience to Chinese visitors. In terms of simple distance and travel times, it is the closest country for those Chinese seeking to experience Western culture. There are also many factors that appeal to the Chinese market including nature and wildlife, iconic attractions, beaches, comfortable climate, and stable politics. Nevertheless, some studies indicate pockets of dissatisfaction with Australia as a travel destination (Commonwealth of Australia, 2005, cited in Breakey et al., 2008; Wang \& Davidson, 2010). In this context, more work that seeks to uncover and understand the various components of their desired Australian experience is well justified.

\section{Approaches to assessing experiences}

Bernd Schmitt, a renowned consultant and marketing thinker, believes that "while no two experiences are exactly the same, experiences can be classified in terms of their generic emerging properties' (Schmitt, 1999, cited in Gretzel \& Fesenmaier, 2010:139). According to Schmitt (2003:105), experiences are based around a meshing of cognitive, sensory, affective, social-identity (or relationship), and physical components. The application of this classification offers promise in assessing the Chinese outbound tourists' experiences. It is recognised that experiences comprise a dynamic mixture of these categories. Food and eating, for example, are mainly sensory experiences but they also have cognitive components (knowing about food), relationship dimensions (one's dining companions) and, further, affective properties since the physical setting for dining can create a distinctive emotional atmosphere. For the sake of not being repetitive, cognitive will refer to mainly cognitive; and this same summary point applies to the other categories. Within this context, a more detailed portrayal of the Schmitt categories can be presented as follows.

Cognitive experiences. Any experience that intellectually stimulates a person can be classed as cognitive. Intellectual and curiosity motives (Agrusa, Kim \& Wang, 2011) and acquiring new knowledge (China Outbound Tourism Research Institute (COTRI), 2009; Zhang \& Lam, 1999) are important factors for Chinese outbound tourists. Chinese travellers also desire to learn from the West and may seek to increase their knowledge about foreign destinations (Xu, Ding \& Packer, 2008; Zhang \& Lam, 1999). Language issues, however, may be an impediment to learning for most Chinese travellers. Those travellers who know English may be shy about using it in case they "lose face" (Briggs, 2006). Similarly, Chinese travellers may be slightly reserved due to their lack of previous travel experience. Any reference to China or Chinese culture whilst abroad would add value to their travel experience (COTRI, 2009). Such references may assist in heightening safety, which is highly valued and constitutes an important destination attribute (Briggs, 2006; Cai, Li \& Knutson, 2007; Li et al., 2011).

Sensory experiences. Experiences that appeal to the five senses are labelled sensory. Sampling local food is an important driver for the Chinese market (Law et al., 2004, cited in Keating \& Kriz, 2008:37). Chinese tourists want food to be a part of the total experience (COTRI, 2009:28; Tourism Queensland, 
2011:43). The opportunity to have familiar Chinese food, however, is also well received (Briggs, 2006; COTRI, 2009:28; Li et al., 2011). Other sensory-related experiences which have been identified as important include getting close to nature (Ai-Ping, 2009), viewing outstanding scenery (COTRI, 2009) and viewing wildlife in natural settings (Tourism Australia, 2004, cited in Weiler and Yu (2006). In addition, Chinese tourists desire a destination with a good climate (Yu \& Weiler, 2001) since pleasant weather offers multiple appealing sensory experiences.

Affective experiences. Experiences which appeal to the customer's inner feelings and emotions are categorised as affective. Very few studies (Ai-Ping, 2009; Weiler \& Yu, 2008) have made specific reference to affective experiences. Despite this, it would appear that Chinese outbound tourists do tend to focus on satisfying their inner needs (Ai-Ping, 2009). For example, the purpose of travel can often be for moral improvement and self-enlightenment (Xu et al., 2008). Furthermore, Chinese tourists believe that travel can provide an escape and an opportunity to relax.

Social-identity (relationship) experiences. Experiences that include social interactions and provide a sense of belonging, and the opportunity to enhance relationships are classified as social-identity. Outbound travel is seen as a mark of sophistication in China, so many Chinese people travel to increase their prestige and self-image (Briggs, 2006; COTRI, 2009; Keating \& Kriz, 2008). As such, visiting famous attractions has become an important part of many Chinese tourists' experience (Zhou et al., 1998). Chinese tourists feel uncomfortable about being alone; consequently they tend to do almost everything together (COTRI, 2009:16). This is quite evident from the domination of group travel and the importance of travelling with friends and family (Keating \& Kriz, 2008; Xu et al., 2008). Similarly, Chinese tourists have a preference for group tours due to safety and convenience (Lo \& Lam, 2004, cited in Keating \& Kriz, 2008:35; Yu \& Weiler, 2001). They also expect tour guides to have a high level of knowledge (Breakey et al., 2008; Li et al., 2011). Chinese travellers also place high demand on guides as temporary persons of authority who can facilitate their experience (COTRI, 2009:17; Weiler and $Y u, 2006)$. A study by Weiler and $\mathrm{Yu}$ (2008) found that Chinese tourists described the opportunity to interact with locals as memorable and important.

Physical experiences, behaviours and lifestyles. Experiences that appeal to behaviours and lifestyles are classed as physical or activity-driven experiences. For Chinese tourists, experiencing Western culture is a priority (Pan \& Laws, 2001). In particular, the opportunities to experience the local lifestyle and reality of living have become major motives. Chinese tourists are also described as looking for new things and something different to their everyday life (Chow\& Murphy, 2011). In addition, shopping is important in their overseas travel experience (COTRI, 2009; Chow \& Murphy, 2011) as gift giving is an essential part of Chinese traditions and relationship management.

The Schmitt categorisation represents one way to conceptualise and assess tourist experiences. Another, and not necessarily incompatible scheme for portraying experiences, lies in the work of Pine and Gilmore (1998), who are well known as pioneers of the experience economy. They suggested that experiences can be categorised depending on the participant's level of involvement (passive-active) and level of connection (absorption-immersion) (Pine \& Gilmore, 1998). The four resultant realms of experiences are entertainment, educational, escapist and aesthetic. Pine and Gilmore's work has been employed in some tourist experience contexts. Its application as a framework for studying the tourist experience has been explored in select tourism venues, including bed-and-breakfast (Oh, Fiore \& Jeoung, 2007), the cruise experience (Hosany \& Witham, 2010), and heritage trails (Hayes \& MacLeod, 2007). In addition, the experience economy concept has been studied as a strategy for destination management (Morgan, Elbe \& de Esteban Curiel, 2009) and for information and communications technology (Stamboulis \& Skayannis, 2003). In all cases, the experience economy concept has been 
recognised as a practical measurement framework for the study of tourist experiences. It can be suggested that for the Chinese outbound market all four realms are important. Entertainment experiences involve passive participation and absorption of an experience such as watching performances and visiting zoos. Chinese outbound tourists have indicated a preference for visiting wildlife parks/zoos/aquariums (Chow \& Murphy, 2008) and a low priority for attending theatre ( $\mathrm{Yu}$ \& Weiler, 2001). With educational experiences, participants require active participation and absorption, such as attending festivals and learning. Visiting historical sites appears to be a priority for Chinese outbound tourists, as identified in several studies (Chow \& Murphy, 2008; Yu \& Weiler, 2001). Activities that require active participation and immersion are classified as escapist. For such activities, shopping is popular (Chow \& Murphy, 2008), whereas dining in Chinese restaurants and attending casinos/gambling are of medium importance, and dining in Western restaurants has low importance (Yu \& Weiler, 2001). Aesthetic experiences involve passive participation and immersion such as viewing landscapes, sightseeing and visiting museums. Chinese outbound tourists do like to visit museums and art galleries (Cai et al., 2007) but give low priority to watching sporting events (Yu \& Weiler, 2001).

\section{Defining the study aims}

The two experience analysis systems reviewed in the preceding section were selected as a focus in this study. Other schemes exist, for some examples see Sundbo and Sorensen (2013), but the prominent approaches being considered deserve research attention, partly as argued in the introduction, because they are so widely cited, but so infrequently compared. In addition, the linking of these two experience analysis systems in a tourism context is clearly warranted, with the potential to explore experiences for many possible combinations of source and destination countries. Specially, the research aims of the study are to:

Aim 1: Explore the perceived importance of Pine and Gilmore's experience realms and Schmitt's categorisations of experience.

Aim 2: Investigate the links between the two experience category systems as revealed by a broad sample of Chinese tourists anticipating a holiday to Australia.

A subsidiary consideration rather than a specific research aim is to use the study to assist in interpreting how to formulate future studies and apply this kind of tourism research.

\section{Methodology}

\section{Research instrument}

To assess the multidimensionality of the anticipated experience, Schmitt's and Pine and Gilmore's categorisation approaches were used. The measures and examples of experiences were built on 20 themes of preferred experiences identified in a detailed literature review (refer to Table 1). Respondents were asked to assess the perceived importance of generic travel versus Australian-specific experiences. This dual questioning was used in an attempt to validate the experience assessment by using two kinds of measures. One can be seen as a priming approach and the other requires more reflection about being immersed in the setting (cf. Mehrabian \& Russell, 1974).

As travel expectations can change with travel, it was important to collect data from mainland Chinese residents before travel. The nature of the research project, however, required respondents to put themselves in a frame of mind about having an Australian experience. In order to do this, an elicitation technique was built into the design of a questionnaire. This approach requires the respondent to pause in the first instance and consider some generic items, and then has the potential to elicit knowledge about more specific and relevant travel domains. It was believed that designing the questionnaire in this way would help respondents to imagine having an Australianfocussed travel experience.

The self-administered questionnaire consisted of two parts. Part A used the generic appraisal questions initially and then a combination of 5point Likert scales and tick-the-box questions to assess the importance levels of the anticipated Australian experience, the desired emotions, and the preferred activities. The range of questions asked permitted the researchers to collect data on both the Pine and Gilmore 
Examining experience economy approaches to tourists' anticipated experiences:

Mainland Chinese travellers consider Australia.

categories and all but one of the elements of the Schmitt approach. Affective experiences were assessed differently to the other Schmitt categories because thinking directly about likely future emotions is a distinctively challenging task. For this component of the Schmitt categories, a more indirect but feasible approach was pilot tested by the researchers and then implemented. Respondents were asked to anticipate what they wanted to feel and achieve from Australian activities and experiences; thus permitting some assumptions about likely emotions. Part B of the questionnaire captured demographic

Table 1. Themes identified in the literature as important for the Chinese market

\begin{tabular}{ll}
\hline Themes & Sub themes \\
\hline \multirow{2}{*}{ Language } & Symbols, signs, brochures, web sites, \\
& $\begin{array}{l}\text { Mandarin-speaking personnel, issue } \\
\text { with communication in English }\end{array}$ \\
Food & $\begin{array}{l}\text { Etiquette, Chinese food, Chinese way, } \\
\text { local }\end{array}$
\end{tabular}

Shopping

Tour guides

Chinese history (connection)

Famous landmarks

Nature/landscapes

Wildlife

Aboriginal culture

Host-guest interaction

Group travel

Australian (western) culture

\section{Education / knowledge}

Creating an atmosphere/ connection feeling

Photos

Escape routine of everyday life

Security

Authenticity

Climate

Status / prestige
For status (luxury), gift-giving, obligation

Chinese, can facilitate experiences Chinese flag, Feng Shui, philosophies, reference to China

Sites, celebrities, attractions

Famous sites in poetry, integration, visiting nature

Status, authority, titles, stronger relationships, "face", honoured guests

Friends and relatives, all-inclusive package tours

Time, experience, historical and cultural sites, cultural interest, differenceforeign cultures;

Connecting sights, stories, poems

Building status, memories Seeing something different, have fun, relaxation, enjoy life, something forbidden

Safety, well-equipped facilities, applying for visa, stable politics

Integration of buildings, spiritual meaning and site

Uniqueness, winter escape

Somewhere friends haven't been; enhancing self-image
Sources

Briggs, 2006; COTRI, 2009; Junek,

Binney and Deery, 2004; Pan and Laws, 2001.

Briggs, 2006; COTRI, 2009; Keating and Kriz, 2008; Zhou, King and Turner, 1998.

Ai-Ping, 2009; Breakey, Ding and Lee, 2008; Cai, Li and Knutson, 2007; COTRI, 2009; Guo, Kim and Timothy, 2007; Junek et al., 2004; Keating and Kriz, 2008; Yu and Weiler, 2001; Zhou et al., 1998.

Junek et al., 2004; Yu and Weiler, 2001.

$\mathrm{Xu}$, Ding and Packer, 2008; COTRI, 2009.

Breakey et al., 2008; Cai et al., 2007;

Keating and Kriz, 2008; Sparks and Pan, 2009; Yu and Weiler, 2001; Zhou et al., 1998.

Breakey et al., 2008; Sparks and Pan, 2009; Xu et al., 2008; Yu and Weiler, 2001.

Breakey et al., 2008.

Breakey et al., 2008; Yu and Weiler, 2001.

Briggs, 2006; COTRI, 2009; Hsu and Lam, 2003; Keating and Kriz, 2008;

Zhang and Lam, 1999.

Keating and Kriz, 2008; Pan and Laws, 2001; Sparks and Pan, 2009; Xu et al., 2008; Zhang and Lam, 1999.

Ai-Ping, 2009; Breakey et al., 2008; Cai et al., 2007; Guo et al., 2007; Li, Xu and Weaver, 2009; Pan and Laws, 2001; Yu and Weiler, 2001.

Breakey et al., 2008; COTRI, 2009; Guo et al., 2007; Hsu and Lam, 2003;

Keating and Kriz, 2008; Li et al., 2009;

Xu et al., 2008; Zhang and Lam, 1999.

COTRI, 2009; Xu et al., 2008.

COTRI, 2009.

Ai-ping, 2009; Briggs, 2006; Cai et al., 2007; Keating and Kriz, 2008; Zhang and Lam, 1999.

Breakey et al., 2008; Keating and Kriz, 2008; Sparks and Pan, 2009; Yu and Weiler, 2001.

Breakey et al., 2008; Li, 2008; Xu et al., 2008.

Breakey et al., 2008.

Ai-Ping, 2009; Breakey et al., 2008; Keating and Kriz, 2008. 
information using tick-the-box options.

A pilot test was conducted in Australia (late June, 2011) to evaluate the logic, clarity and ease of completing the questionnaire. Five Chinese colleagues participated in the pilot study. The questionnaire was translated from English into Mandarin; a process of backtranslation was completed by two James Cook University scholars who are proficient in both languages.

\section{Data collection procedure}

The population for this study was mainland Chinese residents (in one of three regions refer to Figure 1) who have the intention of taking a leisure trip to Australia. Chinese tourists (Hong Kong, Macao and Taiwan travellers) were not selected because this market is different to the mainland Chinese market (Zhou et al., 1998). Furthermore, for historical reasons, citizens of Hong Kong, Macao and Taiwan were considered as different types of outbound tourists (Xie \& Li,

2009). The three regions were chosen because they are the most prosperous economic regions in modern China and are prominent source regions for tourists to Australia (COTRI, 2009:8). Data were collected via the snowball technique during July, 2011. Snowball sampling is a non-random technique that identifies respondents who match a desired profile; then additional respondents are recruited based on the referrals of the original respondents (Black, Asafu-Adjaye, Khan, Perera, Edwards \& Harris, 2007:240).This process involved questionnaires being distributed electronically through the researcher's networks (which had been established through university friends and links) in China, and then their subsequent referrals. The snowball technique was selected as it facilitated ease of data collection and penetration into a population intending to but not yet having travelled (Jackson, White \& Schmierer, 1996, cited in Tung \& Ritchie, 2011:10). Three hundred and thirty-five questionnaires were retrieved. An effective response rate of 95 per cent was achieved as

320 of the 335 completed questionnaires were considered as valid data for further analysis.

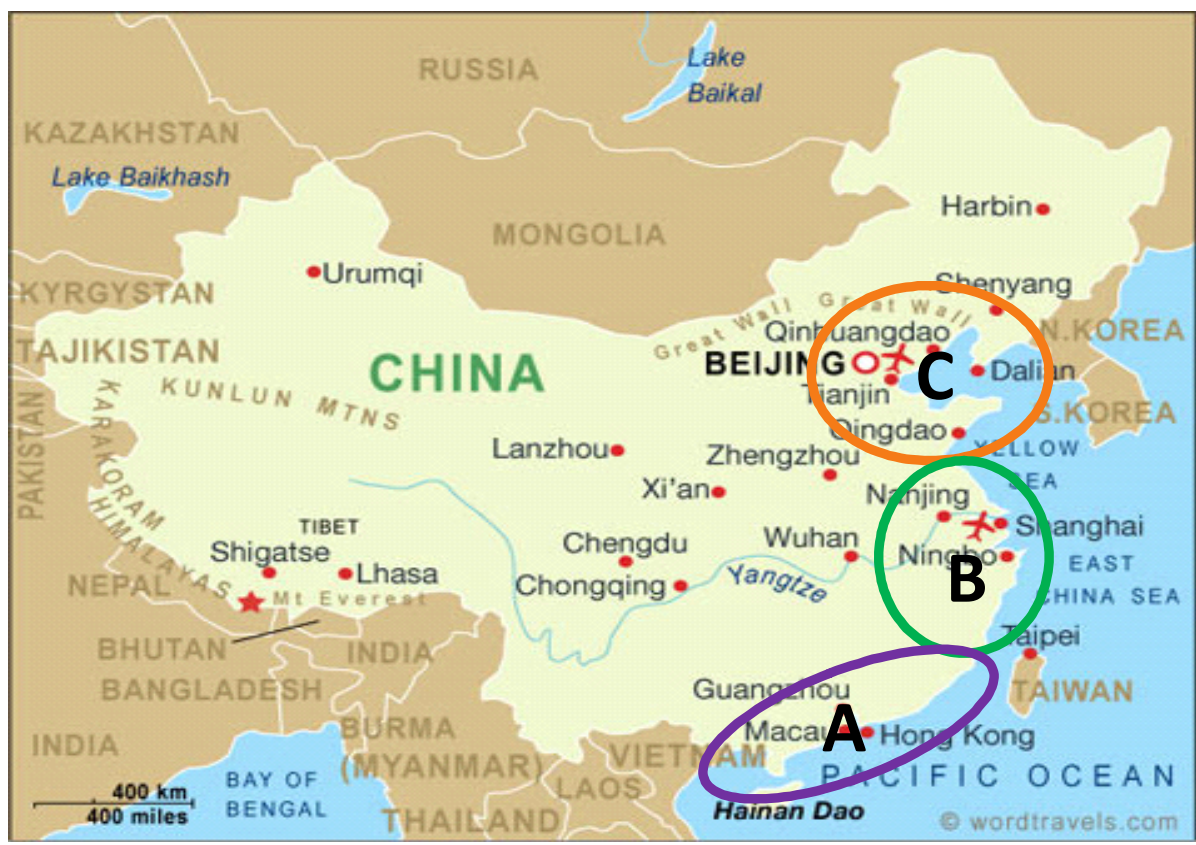

Figure 1.Map of the three regions in China where data were sourced

Note. Regions are based on major cities: A - Guangzhou; B - Shanghai; C - Beijing. 
Demographic profile. The final demographic data set is shown in Table 2. All the demographic questions had a small percentage of respondents who did not specify an appropriate category; these missing percentages are displayed in Table 2. More males participated in the study $(50.4 \%)$ than females $(44.8 \%)$. Most respondents were aged 20-29 years old $(67.8 \%)$. Other respondents were dispersed among the three older age brackets. The sample was geographicallybalanced: region A (31.3\%), region B $(33.4 \%)$, and region C $(30.7 \%)$. Almost every respondent had some domestic travel history, with the majority reporting a medium level of experience $(40.3 \%$ had less than five trips, $34.0 \%$ had between five and 15 trips). Respondents were much less experienced in overseas travel. Over half of the sample $(52.3 \%)$ reported no previous travel with another one-third $(37.0 \%)$ having less than five trips.

\section{Coding and analysis}

The collected data were coded and analysed using SPSS 19.0. Firstly, descriptive statistics (mean, standard deviation, minimum, maximum, kurtosis and skewness) were utilised to assess data entry error and to obtain a quick summary of the characteristics of the variables (Pallant, 2011:43, 53). Data collected on affective experiences and demographics needed no further analysis. Secondly, using the categories developed by Schmitt - effectively an a priori coding scheme - the data from the questionnaires were sorted into the four components of sensory, cognitive, socialidentity and physical. The importance scores for these variables were then computed. Means were then calculated. While data were collected on a 1-5 Likert scale, each new variable (i.e. the importance level of sensory experiences) had five statements that measured it, and therefore, created a possible mean score between five and 25 .

For the Pine and Gilmore categories, new variables built on combinations of items were computed for the importance of entertainment, educational, escapist and aesthetic experiences (four variables in total). The interrelationships among the two schemes were investigated by assessing the four
Schmitt types of experiences within each of the Pine and Gilmore categories. For example, the entertainment variable was segmented into cognitive, sensory, social-identity and physical experiences. Standardised means were used for analysis as there were uneven numbers of variables from the Schmitt categories for each of the Pine and Gilmore classifications.

Table 2. Demographic profile of the sample

\begin{tabular}{|c|c|c|}
\hline & $\mathbf{N}$ & $\%$ \\
\hline \multicolumn{3}{|l|}{ Gender } \\
\hline Male & 169 & 50.4 \\
\hline Female & 150 & 44.8 \\
\hline Missing & 16 & 4.8 \\
\hline \multicolumn{3}{|l|}{ Age } \\
\hline 20-29 years old & 227 & 67.8 \\
\hline $30-39$ years old & 64 & 19.1 \\
\hline $40-49$ years old & 19 & 5.7 \\
\hline 50 years old and older & 9 & 2.7 \\
\hline Missing & 16 & 4.8 \\
\hline \multicolumn{3}{|l|}{ Residence } \\
\hline Region A & 105 & 31.3 \\
\hline Region B & 112 & 33.4 \\
\hline Region C & 103 & 30.7 \\
\hline Missing & 15 & 4.5 \\
\hline \multicolumn{3}{|l|}{ Domestic travel history } \\
\hline Never travelled before & 11 & 3.3 \\
\hline Less than 5 trips & 135 & 40.3 \\
\hline Between 5-15 trips & 114 & 34.0 \\
\hline More than 15 trips & 58 & 17.3 \\
\hline Missing & 17 & 5.1 \\
\hline \multicolumn{3}{|l|}{ Overseas travel history* } \\
\hline Never travelled overseas before & 176 & 52.5 \\
\hline Less than 5 trips & 124 & 37.0 \\
\hline Between 5-15 trips & 15 & 4.5 \\
\hline More than 15 trips & 4 & 1.2 \\
\hline Missing & 16 & 4.8 \\
\hline
\end{tabular}

\section{Results}

The core aims of the study were approached in two parts. The anticipated importance level of each of the Pine and Gilmore experience realms was initially considered. This was assessed by a 5-point Likert scale which asked respondents to indicate their level of anticipated importance for participating in particular activities. Descriptive statistics were utilised to determine the mean anticipated importance level for entertainment, educational, escapist and aesthetic activities. The importance level of all four experience realms was high, with aesthetic scoring the highest $(\mu$ $=36.34)$, followed by educational $(\mu=33.05)$, 
entertainment $(\mu=33.04)$, and escapist $(\mu=$ 31.28).

For the overall scores of the Schmitt categories and anticipated importance levels, no significant differences were reported among physical, social-identity and sensory related experiences. All of these elements were rated with high scores for importance $\left(\mu_{\text {physical }}=\right.$ $\left.19.59 ; \mu_{\text {social-identity }}=19.57 ; \mu_{\text {sensory }}=19.55\right)$. By way of contrast, cognitive experiences were not rated as highly $(\mu=17.12)$. The high levels of these components were not surprising given that Chinese tourists are very curious about Western culture and want to understand different lifestyles, although direct learning and engagement in terms of such themes as Australian history was modest (Agrusa et al., 2011; Pan \& Laws, 2001). The affective experiences were assessed differently to the other Schmitt categorisations. The three highest anticipated emotions were relaxation, joy, and the excitement of discovery. It is clear that Chinese tourists do identify several key emotions as a part of their anticipated experiences (cf. Ai-Ping, 2009).
The relationship between the Pine and Gilmore experience realms and the Schmitt experience categorisations was then able to be built by investigating the means of the Schmitt categories within each of the Pine and Gilmore frameworks. Descriptive statistics were employed to determine the mean anticipated importance level of cognitive, sensory, socialidentity and physical components for each of the Pine and Gilmore realms. The results of this analysis are provided in Figure 2. Within the four realms, physical experiences were the most preferred whereas cognitive experiences were the least. All of the Schmitt experience realms were, however, moderately to highly important for anticipated entertainment, educational, escapist and aesthetic experiences. It was not possible to integrate the affective experiences into these quantitative assessments so the moderating effect of the emotional quality of the experiences needs further research attention in linking the experience economy appraisal systems. Nevertheless, for the emotional perspective, respondents were asked to select all appropriate statements derived from Richins

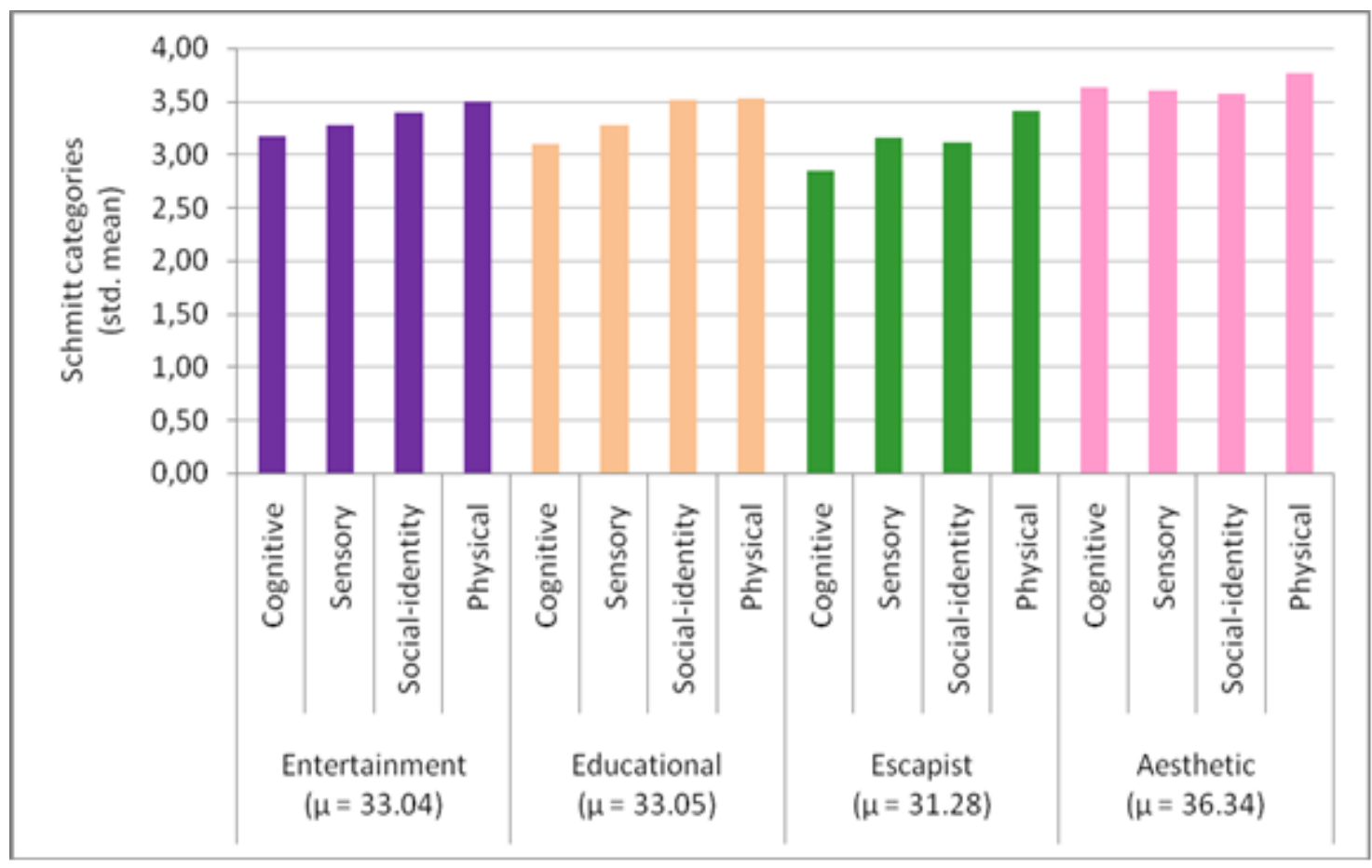

Figure 2.Comparison of Pine and Gilmore and Schmitt anticipated experiences 
Examining experience economy approaches to tourists' anticipated experiences:

Mainland Chinese travellers consider Australia.

Table 3. Preferred affective experiences

\begin{tabular}{llllll}
\hline Emotional statement & $\mathbf{N}$ & $\%$ & Emotional statement & $\mathbf{N}$ & $\%$ \\
\hline A sense of relaxation & 287 & 85.7 & A sense of escape & 110 & 32.8 \\
A sense of joy & 277 & 82.7 & An opportunity to do nothing & 97 & 29.0 \\
An opportunity to discover & 212 & 63.3 & An opportunity to be alone & 66 & 19.7 \\
An opportunity to share & 183 & 54.6 & An opportunity to meet someone (partner) & 37 & 11.0 \\
A sense of excitement & 164 & 49.0 & An opportunity to let out frustration & 32 & 9.6 \\
An opportunity to be thrilled & 144 & 43.0 & A sense of bonding & 27 & 8.1 \\
A sense of fun/happiness & 114 & 34.0 & A sense of accomplishment & 24 & 7.2 \\
An opportunity to be surprised & 111 & 33.1 & A sense of honour/pride & 23 & 6.9 \\
\hline
\end{tabular}

(1997) Consumption Emotion Set. The frequency counts are displayed in Table 3.

The results in Table 3 are dominated by the anticipated emotions of relaxation, joy, discovering, sharing, excited and being thrilled.

\section{Discussion}

The overall objective of this research was to investigate the relationship between Pine and Gilmore's four experience realms of entertainment, educational, escapist and aesthetic and Schmitt's five part categorisation of experiences. Chinese tourists' expectations about Australian travel prior to visits to the destination were used to explore these links. In Figure 2, and using the Pine and Gilmore scores as a first point of focus, respondents indicated a high level of preference for aesthetic experiences over entertainment, educational and escapist activities.

This finding was similar to some previous research. In Pikkemaat, Peters, Boksberger and Secco's (2009) study, aesthetic experiences were rated as the most important and significantly higher than the other experience dimensions for a wine-based touring route experience. Hosany and Witham (2010), in a different application of the experience economy concept, also found that cruisers' experiences can be represented in terms of the four dimensions. In their study aesthetic experiences were again regarded as the highest and escapist experiences the lowest in terms of relative importance. In this work the experience categories were used to explain the outcome variables of memory, arousal, overall perceived quality, satisfaction and intention to recommend. Similarly, Oh et al. (2007) found the aesthetic dimension to be a dominant determinant of the experiential outcomes of bed-and-breakfast experiences, while the escapist and entertainment dimensions were not statistically significant contributors. These results, together with the present work, suggest that all components of the Pine and Gilmore seem to matter and while none are irrelevant to the diverse settings studied in tourism research, there is variability in their importance. The aesthetic component is emerging as a strong candidate to be the most important.

The links between the conceptual schemes can be seen as heightened by the findings and approach adopted in this study. It is apparent to the researchers from the task of measuring the two category schemes that the Pine and Gilmore approach must be assessed, and, is best conceived as operating at a more macro and super-ordinate descriptive level than the Schmitt approach. The Schmitt based categories can be applied both at a generic level and for a highly specific experience level. By embedding the more focussed Schmitt categories within each of the Pine and Gilmore thematic labels some specific links for this sample judging this particular destination were developed. For example, the escapist dimension was seen as the least cognitive and the aesthetic dimension was the most physical. The Schmitt category scores for entertainment experiences were the most varied while aesthetic experiences were uniformly high. This finding suggests that any aesthetic experience is perceived by this market as important whereas certain entertainment experiences are considered to be less important than others. At the specific item level, the results indicated preferences for sightseeing and landscapes rather than engagement in museums, information rich settings and those involving social-identity issues. Similar results were also 
identified in Pikkemaat et al.'s (2009) study where respondents reported a preference for landscapes dominated by wine, but little interest in learning about wine culture (wine and gastronomy) and wine history (wine museums).

This study has also further strengthened the value for conceptual analysis through the use of two widely influential experience analysis schemes. The exploration of the more specific Schmitt categorisations of experience for each of Pine and Gilmore's broad experience realms provided an in-depth understanding of Chinese tourists' wants and needs when considering travel to Australia. As illustrated above, the findings suggest that potential Chinese visitors desire aesthetically - and physically-inclined activities (being immersed in the destination). This is a radical shift from visitors of $10-15$ years ago, and supports the idea that destinations need to understand these changing cultural complexities, as Chinese travellers now expect them to (cf. Li et al., 2011). It will be of significant research interest to begin to explore differences in experiential preferences within the Chinese outbound market, as the emergence of free independent travellers continues to grow alongside of the opening up of travel by citizens of tier two and tier three cities.

The links between the Schmitt and Pine and Gilmore work appear to be sufficiently strong that further work to validate and replicate this first set of connections can be suggested. Nevertheless, as the items were assigned to the Schmitt and Pine and Gilmore components of assessing experiences by the researchers, the results may be seen as subjective. As one attempt to limit this subjectivity, the researchers did agree closely on the placement of the items. Additionally, readers should be aware of the sampling process and actual sample when interpreting results.

The study only surveyed three regions of China and therefore the general nature of the findings is somewhat restricted due to China's large intra-regional differences (Roy, Walters \& Luk, 2001). The tourists' cultural and geographical background can influence many key management and research issues. It can affect tourists' motivations (Zhang \& Lam, 1999), destination decisions ( $\mathrm{Ng}$, Lee \& Soutar, 2007), expectations ( $\mathrm{Li}$ et al., 2011) and the choice of activities ( $\mathrm{Ng}$ et al., 2007). The Chinese leisure market has been described as distinctive because Chinese tourists' expectations, destination choices and behaviours differ from other Asian markets (COTRI, 2009:8; Chow \& Murphy, 2011; Lam \& Hsu, 2004). In reviewing the results of this study there may be special ways in which the Chinese have answered the questions asked. Nevertheless, the choice of this group with their growing importance to global tourism justifies the focus on their anticipated experiences. Checks and different responses to the categories of experience from other markets may however, modify the pattern of content oriented findings reported in the present work.

It is also recognised that the predominantly younger age group in the sample introduce a particular orientation to the data. One main disadvantage of using snowball sampling is that is produces a non-random sample due to respondents being selected based on referral (Black et al., 2007:240). As with many tourism studies, additional samples drawn from the same and different markets would boost the validity of the findings.

While these studies of experience classification continue to evolve and demand further work, it is already clear that a number of marketing organisations use these broad ideas to provide an "experience offer" to tourists (Morrison, 2013). The findings from this study support the idea that marketers could begin to trail the use of the dimensions of the two approaches combined in their presentations. What is less clear is how the categories discussed in this research can be used to shape and evaluate the diversity of these experiential offers in different destination regions, though the core idea of characterizing regional destination personalities through analysing the available experiences is an interesting future tourism research option (Aaker, Kumar \& Day, 1998). Furthermore, the cross-analysis of these two widely influential experience analysis systems could be explored in the future with other target markets and anticipated experiences, as well 
Examining experience economy approaches to tourists' anticipated experiences: Mainland Chinese travellers consider Australia.

as in other tourism experience settings such as cultural- or environmental-based settings.

\section{Conclusions}

There are some positive implications from this study in researching and contemplating tourist experiences. Firstly, this study has suggested that the elicitation technique employed is a workable method to assess the preferred pretravel tourist experience. The findings of the study were comparable to those in the literature thus providing some indirect support for the approach.

Secondly, the results of the study have suggested another potential application of the Pine and Gilmore framework in the assessment of the tourist experience. Previous studies (e.g. Hayes \& MacLeod, 2007; Oh et al., 2007) have applied the framework to study particular tourism spaces e.g. bed-and-breakfast or heritage tourism whereas this study examined a particular tourist type and their desired experience (Chinese outbound tourists considering Australian travel).

A third finding of interest lies in the consideration of the experience preferences identified in the work. It is clear that the cognitive or mentally challenging kinds of involvement provide some difficulties for Chinese tourists accustomed to approaching the world in their own language. At attraction sites, in museums and across the spectrum of interpretation offered to visitors there are issues for outbound Chinese tourists which make thinking about their experience difficult. Recent work suggests this is not simply a matter of language, although the facility in English is important, but also resides in the different cultural lenses and perspectives with which Chinese tourists view the world. Confucian, communist and consumption inspired lenses all represent perspectives which tourism industry personnel might consider to reach their Chinese visitors (Kwek \& Lee, 2010).

A fourth implication from the results suggests that there is the potential to marry the Pine and Gilmore and the Schmitt work in the study of tourist experiences. The fluctuations in the relative importance of the specific Schmitt experience categorisations among the Pine and Gilmore broad experience realms implies that the individual experience components may be viewed differently depending on which kind of broad experience is being considered. Further, conceiving of the Pine and Gilmore work as a broader more overarching framework for representing experiences and the Schmitt work as a flexible system which may be embedded in such larger categories offers a fresh conceptualisation of the intermarriage of these systems.

The possibility of presenting the links between the Schmitt and the Pine and Gilmore approaches in further visual or graphical ways could be explored in future work. Nested or embedded sets of the Schmitt elements distributed across the dimensional framework of the Pine and Gilmore approach might offer the blueprint for this kind of conceptual architecture. Furthermore, future testing of these links could be enhanced by deploying other information collection procedures, including analysing photographs and travellers' blogs, to explore the richness and diversity of the elements considered. Continuing research work on understanding, linking and using experience categories in tourism studies may help inform the increasing use of experientially oriented marketing which is being used by a rising number of destinations.

\section{Acknowledgements}

We thank Huan (Ella) Lu for providing backtranslation to ensure the correctness and clearness of our questionnaires.

\section{References}

Aaker, D.A., Kumar, V. \& Day, G. S. (1998). Marketing research $\left(6^{\text {th }}\right.$ edn). New York: John Wiley \& Sons.

Agrusa, J., Kim, S. S. \& Wang, K-C. (2011). Mainland Chinese tourists to Hawaii: Their characteristics and preferences. Journal of Travel and Tourism Marketing, 28(3), 261278.

Ai-Ping, M. (2009). Motivations for Chinese outbound tourists. The Business Review, Cambridge, 14(1), 150-156.

Black, K., Asafu-Adjaye, J. Khan, N. Perera, N. Edwards, P. \& Harris, M. (2007). Australian Business Statistics. Milton, Australia: John Wiley \& Sons Australia, Ltd. 
Breakey, N., Ding, P. \& Lee, T. (2008). Impact of Chinese outbound tourism to Australia: Reviewing the past; implications for the future. Current Issues in Tourism, 11(6), 587-603.

Briggs, S. (2006). Welcoming Chinese visitors. URL:http://www.multicultural.co.uk/chinese visitors.pdf (Accessed on 23.04.2011).

Cai, L. A., Li, M. \& Knutson, B. J. (2007). Research on China outbound market: A meta-review. Journal of Hospitality and Leisure Marketing, 16(1/2), 5-19.

China Outbound Tourism Research Institute (COTRI) (2009). Are you ready? For Chinese international travellers: A practical guide for delivering superior service. Bangkok, Thailand: Pacific Asia Travel Association (PATA).

Chow, I. \& Murphy, P. (2008). Travel activity preferences of Chinese outbound tourists for overseas destinations. Journal of Hospitality Marketing and Management, 16(1), 61-80.

Chow, I. \& Murphy, P. (2011). Predicting intended and actual travel behaviours: An examination of Chinese outbound tourists to Australia. Journal of Travel and Tourism Marketing, 28(3), 318-330.

Gretzel, U. \& Fesenmaier, D.R. (2010). Capturing sensory experiences through semi-structured elicitation questions. In Morgan, M., P. Lugosi \& Ritchie, J. R. B. (eds) (2010) The tourism and leisure experience: Consumer and managerial perspectives. Bristol, UK: Channel View Publications, 137-160.

Guo, Y., Kim, S. S. \& Timothy, D. J. (2007). Development characteristics and implications of Mainland Chinese outbound tourism. Asia Pacific Journal of Tourism Research, 12 (4), 313-332.

Hayes, D. \& MacLeod, N. (2007). Packaging places: Designing heritage trails using an experience economy perspective to maximise visitor engagement. Journal of Vacation Marketing, 13(1), 45-58.

Hosany, S. \& Witham, M. (2010). Dimensions of cruisers' experiences, satisfaction and intention to recommend. Journal of Travel Research, 49(3), 351-364.

Hsu, C. H. C. \& Lam, T. (2003). Mainland Chinese travelers' motivations and barriers of visiting Hong Kong. Journal of Academy of Business and Economics, 2(1), 60-67.

Huang, S. S. \& Gross, M. J. (2010). Australia's destination image among mainland Chinese travelers: An exploratory study. Journal of Travel and Tourism Marketing, 27(1), 63-81.

Junek, O., Binney, W. \& Deery, M. (2004). Meeting the needs of the Chinese tourist The operator's perspective. ASEAN Journal on Hospitality and Tourism, 3(2), 149-161.

Keating, B. \& Kriz, A. (2008). Outbound tourism from China: Literature review and research agenda. Journal of Hospitality and Tourism Management, 15(1), 32-41.

Kwek, A. \& Lee, Y.S. (2010). Chinese tourists and Confucianism. Asia Pacific Journal of Tourism Research, 15(2), 129-141.

Lam, T. \& Hsu, C. H. C. (2004). Theory of planned behaviour: Potential travelers from China. Journal of Hospitality and Travel Research, 28(4), 463-482.

Li, X., Lai, C. Harrill, R. Kline, S. \& Wang, L. (2011). When east meets west: An exploratory study on Chinese outbound tourists' travel expectations. Tourism Management, 32(4), 741-749.

Li, X., Xu, Y. \& Weaver, P. A. (2009). Motivation segmentation of Chinese tourists visiting the US. Tourism Analysis, 14(4), 515-520.

Mehrabian, A. \& Russell J. A. (1974). An approach to environmental psychology. Cambridge, MA: M.I.T. Press.

Morgan, M., Elbe, J. \& de Esteban Curiel J. (2009). Has the experience economy arrived? The views of destination managers in three visitor-dependent areas. International Journal of Tourism Research, 11(2), 201-216.

Morrison, A. (2013). Marketing and Managing Tourism Destinations. New York: Routledge.

Ng, S. I., Lee, J. A. \& Soutar, G. N. (2007). Tourists' intention to visit a country: The impact of cultural distance. Tourism Management, 28(6), 1497-1506.

Oh, H., Fiore, A. M. \& Jeoung, M. (2007). Measuring experience economy concepts: Tourism applications. Journal of Travel Research, 46(2), 119-132. 
Pallant, J. (2011). SPSS survival manual: A step by step guide to data analysis using SPSS program $\left(4^{\text {th }} e d\right)$. Crows Nest: Allen and Unwin.

Pan, G. W. \& Laws, E. (2001). Tourism marketing opportunities for Australia in China. Journal of Vacation Marketing, 8(1), 39-48.

Pikkemaat, B., Peters, M. Boksberger, P. \& Secco M. (2009). The staging of experiences in wine tourism. Journal of Hospitality Marketing and Management, 18(2-3), 237-253.

Pine, B. J. \& Gilmore, J. H. (1998). Welcome to the experience economy. Harvard Business Review, 76(4), 97-105.

Pine, B. J. \& Gilmore, J. H. (1999). The experience economy: Work is theatre and every business a stage. Boston, Mass: Harvard Business School Press.

Richins, M. L. (1997). Measuring emotions in the consumption experience. The Journal of Consumer Research, 24(2), 127-146.

Roy, A., Walters, P. G. P. \& Luk, S. T. K. (2001). Chinese puzzles and paradoxes: Conducting business research in China. Journal of Business Research, 52(2), 203210.

Schmitt,

B. (1999).

Experiential marketing. Journal of Management Marketing, 15(1-3), 53-67.

Schmitt, B. (2003). Customer experience management: A revolutionary approach to connecting with your customers. Hoboken, New Jersey: John Wiley \& Sons, Inc.

Sparks, B. \& Pan, G. W. (2009). Chinese outbound tourists: Understanding their attitudes, constraints and use of information sources. Tourism Management, 30(4), 483-494.

Stamboulis, Y. \& Skayannis, P. (2003) Innovation strategies and technology for experience-based tourism. Tourism Management, 24(1), 35-43.

Sundbo, J. \& Sorensen, F. (eds) (2013). Handbook on the Experience Economy. Cheltenham: Elgar.

Tourism Queensland (2011). China Strategic Plan 2012-2016. A Discussion Paper. URL: http://www.tq.com.au/fms/tq_corporate/bro chure pdfs/Project\%20China\%20Discussio n\%20Paper\%20October\%202011\%20pdf.p df (Accessed on 16.11.2011).

Tourism Research Australia (2012). Snapshots 2012: China - first and return visitation. URL:http://www.ret.gov.au/tourism/Docume nts/tra/Snapshots\%20and\%20Factsheets/2 012/China_Snapshot_FINAL.pdf

(Accessed on 20.11.2012).

Tung, V. W. S. \& Ritchie, J. R. B. (2011). Exploring the essence of memorable tourism experiences. Annals of Tourism Research, 38(4), 1367-1386.

Wang, Y. \& Davidson, M. C. G. (2010). Preand post-trip perceptions: An insight into Chinese package holiday market to Australia. Journal of Vacation Marketing, 16(2), 111-123.

Weiler, B. \& Yu, X. (2006). Understanding experiences of Chinese visitors to Victoria, Australia. Queensland, Australia: Cooperative Research Centre for Sustainable Tourism.

Weiler, B. \& Yu, X. (2008). Case studies of the experiences of Chinese visitors to three tourist attractions in Victoria, Australia. Annals of Leisure Research, 11(1/2), 225241.

Xie, Y. \& Li, M. (2009). Development of China's outbound tourism and the characteristics of its tourist flow. Journal of China Tourism Research, 5(3), 226-242.

Xu, H., Ding, P. \& Packer, J. (2008). Tourism research in China: Understanding the unique cultural contexts and complexities. Current Issues in Tourism, 11(6), 473-491.

Yu, X. \& Weiler, B. (2001). Mainland Chinese pleasure travelers to Australia: A leisure behaviour analysis. Tourism, Culture and Communication, 3(2), 81-91.

Zhang, H. Q. \& Lam, T. (1999). An analysis of Mainland Chinese visitor's motivations to visit Hong Kong. Tourism Management, 20(5), 587-594.

Zhou, L., King, B. \& Turner, L. (1998). The China outbound market: An evaluation of key constraints and opportunities. Journal of Vacation Marketing, 4(2), 109-119. 